\title{
论 文
}

\section{超硬材料 bc- $\mathrm{B}_{n} \mathrm{CN}(n=1,2,4)$ 的弹性、硬度及其各向 异性}

\author{
胡萌，陈志谦"，李春梅，李凤，王军朋，焦丽娜 \\ 西南大学材料与能源学部, 重庆 400715 \\ *联系人, E-mail: chen_zq@swu.edu.cn \\ 收稿日期: 2015-08-25; 接受日期: 2015-09-29; 网络出版日期: 2016-04-25 \\ 国家重点基础研究发展规划(编号: 2009CB724100)和国家自然科学基金(批准号: 11221062, 11452002)资助项目
}

摘要本文基于密度泛函理论的第一性原理, 分别运用平面波超软噟势和模守恒噟势, 选用局域密度近似 (LDA)和广义梯度近似(GGA)势场计算了所选 3 种结构 $\mathrm{bc}-\mathrm{B}_{n} \mathrm{CN}(n=1,2,4)$ 的弹性和硬度, 并分析了它们的弹性 各向异性和塑性各向异性各自的差异. 结果表明, 3 种结构在热学和力学上均稳定. 能量计算结果显示, B 含量最 低的 bc-BCN 形成焓和结合能绝对值最大, 结构最稳定, 起因于它含有较多能量相对较低的 B-N, B-C, C-N 键及最少的 $\mathrm{B}-\mathrm{B}$ 键. 该 3 种晶体的弹性模量和硬度分析表明, 随着 $\mathrm{B}$ 原子数的增多, 弹性模量和硬度逐渐减小. 根据对普适弹性各向异性指数 $A^{\mathrm{U}}$, 弹性模量各向异性分数比 $A_{\mathrm{B}}, A_{\mathrm{G}}$, 以及剪切模量各向异性因子 $A_{1}, A_{2}$ 和 $A_{3}$ 的 计算, 分析了 3 种结构的弹性各向异性, 结果表明 bc-BCN 具有优于 c-BN 的各向异性性能. 通过对这 3 种结构 进行拉伸和剪切模拟得到理想应力-应变曲线, 发现其形变过程也呈现出各向异性, 指明了不同结构的易屈服面 及滑移方向且 bc- $\mathrm{BCN}$ 是 3 种 $\mathrm{bc}-\mathrm{B}_{n} \mathrm{CN}(n=1,2,4)$ 中最易合成和最适合应用的超硬材料.

关键词第一性原理, 弹性, 硬度, 各向异性

PACS: 71.15.Mb, 46.25.Cc, 62.20.Qp, 62.20.de

\section{1 引言}

近年来, 三元 B-C-N 化合物受到了国内外研究 学者的广泛关注 ${ }^{[1-11]}$. 众所周知, 金刚石是自然界中 最坚硬的固体物质，其硬度可达到 $96 \mathrm{GPa}$. 人工合成 的 c-BN 是硬度仅低于金刚石的超硬材料 $(63 \mathrm{GPa})^{[12]}$. 它们具有相似的物理性质, 如高硬度、高熔点、高耐
磨性等等, 并且已在机械加工、汽车、航空航天等领 域得到广泛应用。

合成超硬材料往往需要高压高温(High Pressure and High Temperature, HPHT)的环境. 高压下物质原 子间距发生变化, Gibbs 自由能亦随之而变, 原子结 构会在另一形式下趋于稳定而形成新的相. 超高压 下物质原子间化学键会发生改变, 并会导致其常规

引用格式: 胡萌, 陈志谦, 李春梅, 等. 超硬材料 $\mathrm{bc}-\mathrm{B}_{n} \mathrm{CN}(n=1,2,4)$ 的弹性、硬度及其各向异性. 中国科学: 物理学 力学 天文学, 2016, 46: 064601 Hu M, Chen Z Q, Li C M, et al. Elasticity, hardness and anisotropies of bc- $\mathrm{B}_{n} \mathrm{CN}(n=1,2,4)$ (in Chinese). Sci Sin-Phys Mech Astron, 2016, 46: 064601, doi: 10.1360/SSPMA2015-00315 
条件下的物理性能，如金属性、超导性、半导体性、 聚合作用、硬度等发生变化. 1981 年, Badzian ${ }^{[13]}$ 在 $\operatorname{HPHT}(14 \mathrm{GPa}, 3300 \mathrm{~K}$ ) 条件下用金刚石和 c-BN 合成 了石墨状的 $\mathrm{B}_{x} \mathrm{C}_{y} \mathrm{~N}_{z}$ 材料. Solozhenko 等人 ${ }^{[14]}$ 也是在 $\operatorname{HPHT}(18 \mathrm{GPa}, 2200 \mathrm{~K})$ 的条件下合成了 $\mathrm{c}-\mathrm{BC}_{2} \mathrm{~N}$, 硬 度达 $76 \mathrm{GPa}$, 高于 $\mathrm{c}-\mathrm{BN}$ 的硬度. Sasaki 等人 ${ }^{[15]}$ 用催化 剂 Co 合成 B-C-N 化合物, 在 $5.5 \mathrm{GPa}, 1400-1600 \mathrm{~K}$ 下, 于熔融的 $\mathrm{Co}$ 催化的 B-C-N 化合物中观察了金刚 石和 c-BN 的瞬时结晶行为. Yao 等人 ${ }^{[16]}$ 通过退火的 无定型状态 B-C-N 粉末, 在 $4 \mathrm{GPa}, 880 \mathrm{~K}$ 条件下, 制 备了一种新的四方 B-C-N 晶体. 但在超高压的极端 条件下, 有些试验价格较高且难以实现, 不利于普遍 应用. 因而, 电子、原子以及分子层次的力学理论模 拟计算分析是研究极端条件下物质微观物理、力学行 为的有力手段, 具有明显的优势. 为了研究金刚石结 构 B-C-N 化合物的电子结构和弹性性质, 研究人员 运用第一性原理计算了 B-C-N 化合物的多种模型. Zhao 等人 ${ }^{[17]}$ 提出了一种 $\mathrm{c}-\mathrm{BC}_{2} \mathrm{~N}$ 无规固溶体模型, 其 中金刚石和 $\mathrm{BN}$ 相的含量基本相当, 改变金刚石和 $\mathrm{BN}$ 的比例时, 计算出的理论硬度在 65-75 GPa 之间 变化. Sun 等人 ${ }^{[18]}$ 研究了 8 原子闪锌矿结构的 $\mathrm{c}-\mathrm{BC}_{2} \mathrm{~N}$, 其中 420 种不同的结构中只有 7 种结构是拓扑不等价 的. 而 Guo 等人 ${ }^{[19]}$ 对这 7 种 $\mathrm{c}-\mathrm{BC}_{2} \mathrm{~N}$ 结构进行计算发 现有四种结构的硬度在 70-72 GPa 之间, 仅次于金刚 石. Zhuang ${ }^{[20]}$ 研究了 $\mathrm{c}-\mathrm{BC}_{\mathrm{y}} \mathrm{N}$ 材料的结构稳定性、力 学性质、电子结构等等, 发现和 $\mathrm{BC}_{2} \mathrm{~N}$ 相比, 随着 $\mathrm{C}(y>2)$ 含量的升高它有更稳定的结构以及更高的弹 性模量. Komatsu 等人 ${ }^{[21]}$ 利用冲击压缩法制备了 $\mathrm{c}-\mathrm{BC}_{\mathrm{y}} \mathrm{N}(y=1,2,7-11)$, 这些合成的 $\mathrm{c}-\mathrm{BC}_{y} \mathrm{~N}$ 都具有与 c-BN 相近的硬度.

从当前的研究现状来看, 我们不难发现, 在理论 和实验中, $\mathrm{BC}_{y} \mathrm{~N}$ 化合物 (特别是 $y=2-5$ 时)研究较为成 熟, 而对于 $\mathrm{B}_{n} \mathrm{CN}$ 的研究较少, 直到近几年才有人关 注 $\mathrm{B}_{2} \mathrm{CN}$ 材料. $\mathrm{He}$ 等人 ${ }^{[22]}$ 在 $5.5 \mathrm{GPa}$ 高压和 $1600^{\circ} \mathrm{C}$ 下合成了正交 $\beta-\mathrm{B}_{2} \mathrm{CN}\left(\mathrm{B}_{0.47} \mathrm{C}_{0.23} \mathrm{~N}_{0.30}\right)$, 这种材料和金 刚石比起来密度较小且具有良好的发光性能. $\mathrm{Li}$ 等 人 ${ }^{[23]}$ 对 5 类金刚石结构的 $\mathrm{B}_{2} \mathrm{CN}$ 进行理论计算, 发现 它们有较大的体弹模量(333 GPa)和硬度(56-58 GPa), 并且呈现出超导特性. 当 $\mathrm{C}$ 原子的比例改变时, 研究 学者们发现材料的性能有意想不到的变化, 但 B 含 量对于材料整体性能的影响鲜有系统研究. 因此, 我
们根据 $\mathrm{C}_{24}$ 构造了 3 类结构. 与原超硬材料结构相比, 它们呈现出独特的原子分布, 晶胞中所有原子都可 呈现于晶胞表面, 即原子会形成蜂巢状的排布 ${ }^{[24,25]}$. 这种有望实现高比强度的超硬材料表现出的特有性 质是我们进行研究的目的. 本文采用基于密度泛函 的第一性原理平面波超软赝势和模守恒赝势, 运用 局域密度近似(LDA)和广义梯度近似(GGA)势场进行 计算, 分析了 3 种正交结构的弹性、硬度及各向异性 特征, 为得到综合性能更加优越的新型超硬材料和 实现非 HPHT 合成提供理论依据.

\section{2 计算参数与理论}

\section{1 计算模型}

本文所研究的 3 种 bc- $\mathrm{BCN}, b c-\mathrm{B}_{2} \mathrm{CN}, \mathrm{bc}-\mathrm{B}_{4} \mathrm{CN}$ 正交晶系结构模型源自 $\mathrm{C}_{24}$ 模型. 将其 $\{100\},\{010\}$ 和 $\{001\}$ 方向上的 C 原子分别移至(100), (010)和(001) 面上, 再分别由 $\mathrm{B}$ 原子和 $\mathrm{N}$ 原子替代, 如图 1 所示, 空间群为 Pmm2(Space group No.25). Luo 等人 ${ }^{[24]}$ 和 Ribeiro 等人 ${ }^{[25]}$ 先后搭建了空间群为 $I m-3 m$ (Space group No.229)的 bc6-C 体心立方结构, 并且利用该结 构构建了图 1 所示的类似结构. 首先我们搭建了不同 拓扑结构的 bc- $\mathrm{B}_{n} \mathrm{CN}(n=1,2,4)$, 然后对相同元素比 例含量、不同占位的 $\mathrm{bc}-\mathrm{B}_{n} \mathrm{CN}$ 进行结构优化, 确定不 同占位的体系能量. 并进一步选定 $n$ 在不同取值下的 体系能量较低的和各向异性较小的结构, 如图 1 所 示. 本文计算单元为一个原胞, 每个晶胞均含有 12 个原子.

\section{2 计算公式}

体系的总能量可以表示为对微小应变 $\delta$ 的泰勒 级数展开形式

$$
E(V, \delta)=E\left(V_{0}, 0\right)+V_{0}\left[\sum_{i} \tau_{i} \delta_{i}+\frac{1}{2} \sum_{i j} \tau_{i j} \varepsilon_{i j} \delta_{i j}\right],
$$

其中, $E\left(V_{0}, 0\right)$ 和 $V_{0}$ 是未施加应变时体系的总能量和 体积, $\tau_{i}$ 是应力张量元, 而 $\varepsilon_{i}$ 是 Voigt 因子.

弹性常数 $C_{i j}$ 可表示为系统总能量对应变的二阶 导数

$$
C_{i j}=\frac{\mathrm{d} E}{\mathrm{~d} \delta} .
$$

对于正交晶系, 共有 9 个独立的不为零的矩阵元 
(a)

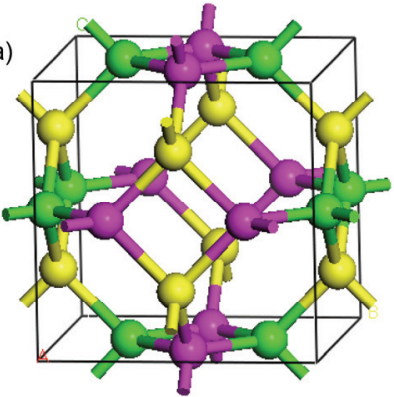

(b)

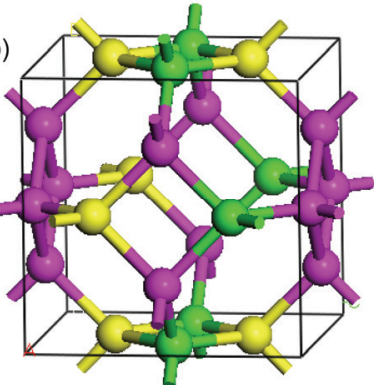

(c)

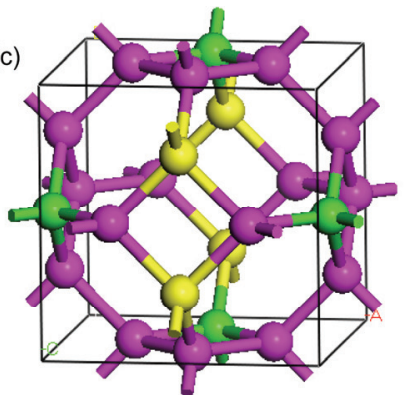

图 1 (网络版彩图) 3 种 bc- $\mathrm{B}_{n} \mathrm{CN}(n=1,2,4)$ 晶体结构示意图: (a) bc-BCN; (b) bc- $\mathrm{B}_{2} \mathrm{CN}$; (c) bc- $\mathrm{B}_{4} \mathrm{CN}$. $\bullet \mathrm{B}$ 原子, $\circ \mathrm{C}$ 原子, $\mathrm{N}$ 原子

Figure 1 (Color online) Schematic diagram of bc- $\mathrm{B}_{n} \mathrm{CN}(n=1,2,4)$ crystal structure: (a) bc-BCN; (b) bc- $\mathrm{B}_{2} \mathrm{CN}$; (c) bc- $\mathrm{B}_{4} \mathrm{CN}$. $\bigcirc \mathrm{B}$ atom, $\mathrm{C}$ atom, $\mathrm{N}$ atom.

$$
C_{i j}=\left[\begin{array}{cccccc}
C_{11} & C_{12} & C_{13} & 0 & 0 & 0 \\
C_{21} & C_{22} & C_{23} & 0 & 0 & 0 \\
C_{31} & C_{32} & C_{33} & 0 & 0 & 0 \\
0 & 0 & 0 & C_{44} & 0 & 0 \\
0 & 0 & 0 & 0 & C_{55} & 0 \\
0 & 0 & 0 & 0 & 0 & C_{66}
\end{array}\right],
$$

与之相对应的顺度矩阵为

$$
S_{i j}=\left[\begin{array}{cccccc}
S_{11} & S_{12} & S_{13} & 0 & 0 & 0 \\
S_{21} & S_{22} & S_{23} & 0 & 0 & 0 \\
S_{31} & S_{32} & S_{33} & 0 & 0 & 0 \\
0 & 0 & 0 & S_{44} & 0 & 0 \\
0 & 0 & 0 & 0 & S_{55} & 0 \\
0 & 0 & 0 & 0 & 0 & S_{66}
\end{array}\right],
$$

对于多晶系, Hill ${ }^{[26]}$ 证明 VRH (Voigt-Reuss-Hill)与实 验结果更为接近

$$
\begin{aligned}
& B=\frac{1}{2}\left(B_{\mathrm{V}}+B_{\mathrm{R}}\right), \\
& G=\frac{1}{2}\left(G_{\mathrm{V}}+G_{\mathrm{R}}\right),
\end{aligned}
$$

式中 $B_{\mathrm{V}}, G_{\mathrm{V}}$ 分别为 Voigt 模型体弹模量和剪切模量, $B_{\mathrm{R}}, G_{\mathrm{R}}$ 分别为 Reuss 模型体弹模量和剪切模量, 其公 式 ${ }^{[27,28]}$ 如下:

$$
\begin{aligned}
B_{\mathrm{V}}= & \frac{1}{9}\left[\left(C_{11}+C_{22}+C_{33}\right)+2\left(C_{12}+C_{13}+C_{23}\right),\right. \\
G_{\mathrm{V}}= & \frac{1}{15}\left[\left(C_{11}+C_{22}+C_{33}\right)-\left(C_{12}+C_{13}+C_{23}\right)\right. \\
& \left.+\left(C_{44}+C_{55}+C_{66}\right)\right],
\end{aligned}
$$

$$
\begin{aligned}
& \quad B_{\mathrm{R}}=\frac{1}{\left[\left(S_{11}+S_{22}+S_{33}\right)+2\left(S_{12}+S_{13}+S_{23}\right)\right]}, \\
& G_{\mathrm{R}}= \\
& \frac{15}{\left[4\left(S_{11}+S_{22}+S_{33}\right)-4\left(S_{12}+S_{13}+S_{23}\right)+3\left(S_{44}+S_{55}+S_{66}\right)\right]} .
\end{aligned}
$$

通过计算体弹模量和剪切模量的 Hill值，我们进 一步得到了多晶体系下各材料的杨氏模量和泊松比

$$
\begin{gathered}
E=\frac{9 B G}{3 B+G}, \\
v=\frac{3 B-2 G}{2(3 B+G)} .
\end{gathered}
$$

材料的德拜温度由下式计算求得 ${ }^{[29]}$

$$
\Theta_{\mathrm{D}}=\frac{h}{k}\left[\frac{3 n}{4 \pi}\left(\frac{N_{\mathrm{A}} \rho}{M}\right)\right]^{1 / 3} v_{\mathrm{m}},
$$

式中 $v_{\mathrm{m}}$ 代表平均声学波波速, $v_{\mathrm{m}}{ }^{[30]}$ 的公式为

$$
v_{\mathrm{m}}=\frac{1}{3}\left[\frac{2}{v_{\mathrm{t}}^{3}}+\frac{1}{v_{1}^{3}}\right]^{-1 / 3},
$$

$v_{\mathrm{t}}$ 和 $v_{1}$ 表示平均声学横波和声学纵波波速, 其公式分 别如下

$$
\begin{gathered}
v_{\mathrm{t}}=\sqrt{G / \rho}, \\
v_{1}=\sqrt{(\mathrm{B}+4 G / 3) / \rho} .
\end{gathered}
$$

\section{3 计算参数}

本文计算采用基于密度泛函理论(DFT) ${ }^{[31]}$ 的第 一性原理方法, 由 $\mathrm{CASTEP}^{[32]}$ 程序完成. 计算过程采 用周期性边界条件, 对交换关联势能采用了局域密 度近似(LDA)下的 CA-PZ 方法 ${ }^{[33]}$ 和广义梯度近似 
(GGA)下的 Perdew-Burke-Ernzerhof (PBE) 方 法 $^{[34]}$, 晶体波函数由平面波基矢展开，并由超软噟势 (Ultrasoft Pseudopotential, USPP) 和模守恒噟势 (Norm-Conserving Pseudopetential, NCPP) ${ }^{[35]}$ 来实现 离子实与价电子间的相互作用势. 局域密度近似 (LDA)假定了体系中的原子核间距较远, 电子在其晶 格背景中的运动可以近似认为是均匀场中的行为, 适用于各种体系基态性质的计算, 而且在对一些体 系的弹性性质、状态方程以及在高温高压下的相变等 方面进行计算时都取得很好的结果.广义梯度近似 (GGA)引入了电荷梯度来修正电荷密度的局域变化, 并较大幅度地修正了少电荷密度区域的指数形式, 在结构、总能、带隙等的计算中和实验结果符合较好. 超软赝势(USPP)引入了广义的正交归一化条件, 使 得波函数变得更平滑, 所需的平面波基底函数更少, 仅需要非常小的截断能. 模守恒噟势 (NCPP) 的准确 性要求较高的截断能量, 能产生正确的电荷密度, 适 合作自洽计算 ${ }^{[36]}$.

原子噟势计算考虑的外层电子组态为: $\mathrm{B}$ 为 $2 s^{2} 2 p^{1}, C$ 为 $2 s^{2} 2 p^{2}, N$ 为 $2 s^{2} 2 p^{3}$. 根据收玫性测试, 我 们选择波矢 $\mathrm{K}$ 空间中 bc-BCN, bc- $\mathrm{B}_{2} \mathrm{CN}, \mathrm{bc}-\mathrm{B}_{4} \mathrm{CN}$ 的 平面波截断能 $780 \mathrm{eV}$. 根据收玫性测试, 其布里渊区 积分计算分别为 $6 \times 6 \times 6,10 \times 10 \times 10,10 \times 10 \times 10$ 的 Monkors-Park ${ }^{[37]}$ 特殊 $\mathrm{K}$ 点对全布里洲区求和. 当系统 总能量变化稳定在 $5 \times 10^{-6} \mathrm{eV}$ 以内, 并使得优化后作 用在晶胞中每个原子上的力小于 $0.01 \mathrm{eV} / \AA$, 晶胞剩 余应力低于 $0.02 \mathrm{GPa}$, 公差偏移小于 $5 \times 10^{-4} \AA$, 认为 达到收玫. 结构优化计算所使用的晶格常数为实验 值, 运用 BFGS 算法 ${ }^{[38-41]}$ 先后对晶体模型结构及晶格 原子的位置进行优化, 以找出能量最低的结构, 并在 此基础上进一步计算其弹性性质、硬度及各向异性.

\section{3 计算结果与讨论}

\section{1 晶体结构的稳定性分析}

为了获得 $\mathrm{bc}-\mathrm{B}_{n} \mathrm{CN}$ 的基态晶格参量, 确定晶格 常数 $a, b$ 和 $c$, 本文先对晶胞进行结构优化, 获得基 态下的能量. 晶胞的体积优化采用非自旋极化方式, 以确定基态能量, 进而获得基态的体积(晶格常数)和 体积模量 $B$. 体积模量 $B$ 定义为

$$
B=-\frac{\partial P}{\partial V}=V \frac{\partial^{2} E}{\partial V^{2}},
$$

式中 $E$ 为基态的总能, $P$ 和 $V$ 为压强和体积. 体积优 化是计算 $\mathrm{bc}-\mathrm{B}_{n} \mathrm{CN}$ 不同体积时的总能. 根据下列 Brich-Mumaghan ${ }^{[42]}$ 状态方程可确定体积与总能之间 的关系

$$
\begin{aligned}
E(V)= & E_{0}+\frac{9 V_{0} B_{0}}{16}\left\langle\left[\left(\frac{V_{0}}{V}\right)^{2 / 3}-1\right]^{3} B_{0}^{\prime}\right. \\
& \left.+\left[\left(\frac{V_{0}}{V}\right)^{2 / 3}-1\right]^{2}\left[6-4\left(\frac{V_{0}}{V}\right)^{2 / 3}\right]\right\rangle,
\end{aligned}
$$

式中 $E_{0}$ 为基态总能, $V_{0}$ 为基态体积, $B_{0}$ 和 $B_{0}^{\prime}$ 分别是体 积模量和零压下的体积模量对压强的导数.

计算优化得到的晶格参数、体积、密度、单原子 能 $E_{\mathrm{a}}$ 以及键的个数、形成焓 $\Delta H$ 和结合能 $\Delta E_{\mathrm{coh}}$ 如表 1 所示. 其中, 形成焓和结合能的公式如下所示 ${ }^{[43]}$ : 形成焓

$$
\Delta H=\frac{1}{n+2}\left(E_{\mathrm{tot}}-n E_{\text {solid }}^{\mathrm{B}}-E_{\text {solid }}^{\mathrm{C}}-E_{\text {solid }}^{\mathrm{N}}\right),
$$

结合能

$$
\Delta E_{\text {coh }}=\frac{1}{n+2}\left(E_{\text {tot }}-n E_{\text {atom }}^{\mathrm{B}}-E_{\text {atom }}^{\mathrm{C}}-E_{\text {atom }}^{\mathrm{N}}\right),
$$

形成焓公式中 $E_{\mathrm{tot}}, E_{\text {solid }}^{\mathrm{B}}, E_{\mathrm{solid}}^{\mathrm{C}}$ 和 $E_{\mathrm{solid}}^{\mathrm{N}}$ 分别代表本 文计算所得 $\mathrm{bc}-\mathrm{B}_{n} \mathrm{CN}$ 的计算单元总能量以及单质 $\mathrm{B}$, $\mathrm{C}, \mathrm{N}$ 结构晶胞中单个原子的能量; 结合能公式中 $E_{\text {atom }}^{\mathrm{B}}, E_{\text {atom }}^{\mathrm{C}}, E_{\text {atom }}^{\mathrm{N}}$ 则分别代表 $\mathrm{B}, \mathrm{C}$ 和 $\mathrm{N}$ 原子的单原 子能量.

我们从表 1 中可以看出, bc- $\mathrm{B}_{n} \mathrm{CN}(n=1,2,4)$ 的结 合能都为负数, 并且它们的能量按 $n=1,2,4$ 的顺序逐 渐增大, 可以确定 3 种结构中 bc-BCN 最稳定. 由表 1 中原子键的成键数量可以看出, bc-BCN 中所含的 $\mathrm{B}-\mathrm{N}$ 键较多, 降低了系统的总能量, 而 $b c-\mathrm{B}_{2} \mathrm{CN}$ 虽 然和 bc-BCN 中所含 $\mathrm{B}-\mathrm{C}$ 键数目相同, 但是其含有 $\mathrm{B}-\mathrm{B}$ 键替代了较低能量的键, 使得体系总能量上升. bc- $\mathrm{B}_{4} \mathrm{CN}$ 中含有最多的 $\mathrm{B}-\mathrm{B}$ 键和较少的 $\mathrm{B}-\mathrm{N}$, $\mathrm{B}-\mathrm{C}, \mathrm{C}-\mathrm{N}$ 键, 对应最高的体系总能量. 形成焓是 不同类原子从其单质状态生成化合物所释放或吸收 的能量, 它表示化合物生成的难易程度, 当形成焓为 负时, 其绝对值越大, 化合物越容易形成; 晶体的结 合能是自由原子结合为晶体所释放的能量, 晶体的 强度、结构稳定性与其结合能密切相关, 绝对值越大, 晶体就越稳定 ${ }^{[43]}$. 我们计算所得的形成焓和结合能 
同为负数, 这说明 $\mathrm{bc}-\mathrm{B}_{n} \mathrm{CN}(n=1,2,4)$ 的 3 种结构模 型在热学上都是稳定的, 其中 bc-BCN 最容易合成且 结构最为稳定.

表 2 所示为 $\mathrm{bc}-\mathrm{B}_{n} \mathrm{CN}(n=1,2,4)$ 的弹性常数, 弹
性常数表征了晶格在弹性限度内对外应力作用的反 应. 由表 1 和 2 数据可知, 利用 LDA 势场优化所得的 晶格常数较小, 对应的弹性常数较大, 而 GGA 势场 所产生的结论正好相反; 超软赝势和模守恒赝势

表 $1 \mathrm{bc}-\mathrm{B}_{n} \mathrm{CN}(n=1,2,4) 3$ 种结构模型的晶格参数、体积、密度、单原子能 $E_{\mathrm{a}}(\mathrm{eV} / \mathrm{atom}) 、$ 形成热 $\Delta H(\mathrm{eV}) 、$ 结合能 $\Delta E_{\mathrm{coh}}(\mathrm{eV})$ 和键的个数. 表中(1)代表超软赝势; (2)代表模数守恒势

Table 1 Lattice constants $a, b, c(\AA)$, volume $V\left(\AA^{3}\right)$, density $\rho\left(\mathrm{g} \cdot \mathrm{cm}^{-3}\right), E_{\mathrm{a}}(\mathrm{eV} / \mathrm{atom}), \Delta H(\mathrm{eV}), \Delta E_{\text {coh }}(\mathrm{eV})$ and bond number of bc- $\mathrm{B}_{n} \mathrm{CN}(n=1,2,4)$. (1): Ultrasoft; (2): norm-conserving

\begin{tabular}{|c|c|c|c|c|c|c|c|}
\hline & & \multicolumn{2}{|c|}{$\mathrm{BCN}$} & \multicolumn{2}{|c|}{$\mathrm{B}_{2} \mathrm{CN}$} & \multicolumn{2}{|c|}{$\mathrm{B}_{4} \mathrm{CN}$} \\
\hline & & LDA & GGA & LDA & GGA & LDA & GGA \\
\hline \multirow[b]{2}{*}{$a$} & (1) & 4.386 & 4.444 & 4.590 & 4.639 & 3.930 & 4.031 \\
\hline & (2) & 4.401 & 4.460 & 4.607 & 4.658 & 3.944 & 4.041 \\
\hline \multirow{2}{*}{$b$} & (1) & 4.385 & 4.443 & 4.024 & 4.110 & 4.567 & 4.604 \\
\hline & (2) & 4.401 & 4.460 & 4.032 & 4.116 & 4.574 & 4.617 \\
\hline \multirow[b]{2}{*}{$c$} & (1) & 4.365 & 4.427 & 4.701 & 4.748 & 5.071 & 5.124 \\
\hline & (2) & 4.387 & 4.448 & 4.711 & 4.758 & 5.080 & 5.132 \\
\hline \multirow{2}{*}{$V$} & (1) & 83.948 & 87.411 & 86.823 & 90.514 & 91.013 & 95.092 \\
\hline & (2) & 84.954 & 88.467 & 87.511 & 91.216 & 91.628 & 95.727 \\
\hline$\rho$ & (1) & 2.931 & 2.814 & 2.749 & 2.637 & 2.542 & 2.433 \\
\hline$E_{\mathrm{a}}$ & (1) & -168.174 & -167.672 & -145.590 & -145.094 & -122.762 & -122.278 \\
\hline$\Delta H$ & (1) & -1.069 & -0.758 & -0.844 & -0.618 & -0.375 & -2.240 \\
\hline$\Delta E_{\mathrm{coh}}$ & (1) & -8.984 & -8.036 & -8.651 & -7.753 & -8.075 & -7.753 \\
\hline \multicolumn{2}{|c|}{$\mathrm{B}-\mathrm{N}$ bond } & \multicolumn{2}{|c|}{8} & \multicolumn{2}{|c|}{8} & \multicolumn{2}{|c|}{6} \\
\hline \multicolumn{2}{|c|}{$\mathrm{B}-\mathrm{C}$ bond } & \multicolumn{2}{|c|}{8} & \multicolumn{2}{|c|}{8} & \multicolumn{2}{|c|}{6} \\
\hline \multicolumn{2}{|c|}{$\mathrm{C}-\mathrm{N}$ bond } & \multicolumn{2}{|c|}{8} & \multicolumn{2}{|c|}{4} & \multicolumn{2}{|c|}{2} \\
\hline \multicolumn{2}{|c|}{ B-B bond } & \multicolumn{2}{|c|}{-} & \multicolumn{2}{|c|}{4} & \multicolumn{2}{|c|}{10} \\
\hline
\end{tabular}

表 2 bc- $\mathrm{B}_{n} \mathrm{CN}(n=1,2,4) 3$ 种结构模型的弹性刚度矩阵常数(GPa). 表中(1)代表超软赝势; (2)代表模数守恒势

Table 2 Elastic stiffness constants (GPa) of the three bc- $\mathrm{B}_{n} \mathrm{CN}(n=1,2,4)$. (1): Ultrasoft; (2): norm-conserving

\begin{tabular}{|c|c|c|c|c|c|c|c|}
\hline & & \multicolumn{2}{|c|}{$\mathrm{BCN}$} & \multicolumn{2}{|c|}{$\mathrm{B}_{2} \mathrm{CN}$} & \multicolumn{2}{|c|}{$\mathrm{B}_{4} \mathrm{CN}$} \\
\hline & & LDA & GGA & LDA & GGA & LDA & GGA \\
\hline$C_{11}$ & $\begin{array}{l}\text { (1) } \\
\text { (2) }\end{array}$ & $\begin{array}{l}655 \\
643\end{array}$ & $\begin{array}{l}608 \\
597\end{array}$ & $\begin{array}{l}590 \\
585\end{array}$ & $\begin{array}{l}541 \\
536\end{array}$ & $\begin{array}{l}267 \\
264\end{array}$ & $\begin{array}{l}242 \\
240\end{array}$ \\
\hline$C_{22}$ & $\begin{array}{l}\text { (1) } \\
\text { (2) }\end{array}$ & $\begin{array}{l}655 \\
643\end{array}$ & $\begin{array}{l}608 \\
597\end{array}$ & $\begin{array}{l}322 \\
330\end{array}$ & $\begin{array}{l}310 \\
309\end{array}$ & $\begin{array}{l}376 \\
372\end{array}$ & $\begin{array}{l}353 \\
349\end{array}$ \\
\hline$C_{33}$ & $\begin{array}{l}\text { (1) } \\
\text { (2) }\end{array}$ & $\begin{array}{l}730 \\
722\end{array}$ & $\begin{array}{l}705 \\
696\end{array}$ & $\begin{array}{l}877 \\
868\end{array}$ & $\begin{array}{l}834 \\
816\end{array}$ & $\begin{array}{l}774 \\
763\end{array}$ & $\begin{array}{l}728 \\
717\end{array}$ \\
\hline$C_{44}$ & $\begin{array}{l}\text { (1) } \\
\text { (2) }\end{array}$ & $\begin{array}{l}220 \\
214\end{array}$ & $\begin{array}{l}209 \\
203\end{array}$ & $\begin{array}{l}142 \\
138\end{array}$ & $\begin{array}{l}137 \\
134\end{array}$ & $\begin{array}{l}120 \\
110\end{array}$ & $\begin{array}{l}116 \\
114\end{array}$ \\
\hline$C_{55}$ & $\begin{array}{l}\text { (1) } \\
\text { (2) }\end{array}$ & $\begin{array}{l}220 \\
217\end{array}$ & $\begin{array}{l}209 \\
203\end{array}$ & $\begin{array}{l}195 \\
193\end{array}$ & $\begin{array}{l}187 \\
185\end{array}$ & $\begin{array}{l}50 \\
53\end{array}$ & $\begin{array}{l}64 \\
52\end{array}$ \\
\hline$C_{66}$ & $\begin{array}{l}\text { (1) } \\
\text { (2) }\end{array}$ & $\begin{array}{l}211 \\
205\end{array}$ & $\begin{array}{l}195 \\
189\end{array}$ & $\begin{array}{l}151 \\
151\end{array}$ & $\begin{array}{l}151 \\
151\end{array}$ & $\begin{array}{l}107 \\
109\end{array}$ & $\begin{array}{l}110 \\
111\end{array}$ \\
\hline$C_{12}$ & $\begin{array}{l}\text { (1) } \\
\text { (2) }\end{array}$ & $\begin{array}{l}140 \\
132\end{array}$ & $\begin{array}{l}122 \\
115\end{array}$ & $\begin{array}{l}184 \\
176\end{array}$ & $\begin{array}{l}174 \\
166\end{array}$ & $\begin{array}{l}202 \\
196\end{array}$ & $\begin{array}{l}187 \\
182\end{array}$ \\
\hline$C_{13}$ & $\begin{array}{l}\text { (1) } \\
\text { (2) }\end{array}$ & $\begin{array}{l}91 \\
84\end{array}$ & $\begin{array}{l}68 \\
62\end{array}$ & $\begin{array}{l}84 \\
79\end{array}$ & $\begin{array}{l}71 \\
66\end{array}$ & $\begin{array}{l}48 \\
46\end{array}$ & $\begin{array}{l}44 \\
42\end{array}$ \\
\hline$C_{23}$ & $\begin{array}{l}\text { (1) } \\
\text { (2) }\end{array}$ & $\begin{array}{l}92 \\
84\end{array}$ & $\begin{array}{l}68 \\
62\end{array}$ & $\begin{array}{l}82 \\
79\end{array}$ & $\begin{array}{l}72 \\
70\end{array}$ & $\begin{array}{l}116 \\
107\end{array}$ & $\begin{array}{l}93 \\
88\end{array}$ \\
\hline$C_{44} / C_{66}$ & $\begin{array}{l}\text { (1) } \\
\text { (2) }\end{array}$ & $\begin{array}{l}1.043 \\
1.044\end{array}$ & $\begin{array}{l}1.072 \\
1.074\end{array}$ & $\begin{array}{l}0.940 \\
0.914\end{array}$ & $\begin{array}{l}0.907 \\
0.887\end{array}$ & $\begin{array}{l}1.121 \\
1.009\end{array}$ & $\begin{array}{l}1.055 \\
1.027\end{array}$ \\
\hline$C_{55} / C_{66}$ & $\begin{array}{l}1 \\
\text { (2) }\end{array}$ & $\begin{array}{l}1.043 \\
1.058\end{array}$ & $\begin{array}{l}1.072 \\
1.074\end{array}$ & $\begin{array}{l}1.291 \\
1.261\end{array}$ & $\begin{array}{l}1.238 \\
1.225\end{array}$ & $\begin{array}{l}0.467 \\
0.486\end{array}$ & $\begin{array}{l}0.582 \\
0.468\end{array}$ \\
\hline
\end{tabular}


计算也具有一致的规律, 利用超软㕍势所得参数要 大于模守恒噟势计算所得的. 然而不论利用哪一种 势场或噟势来优化晶胞, 其计算数据所具有的趋势 一致, 故以后只列出超软噟势下 LDA 算法给出的 数据.

对于正交晶系, 其力学稳定性判据为 ${ }^{[44]}$

$$
\begin{gathered}
C_{i i}>0,(i, j=1-6), \quad\left[C_{11}+C_{22}+C_{33}+2\left(C_{12}+C_{13}+C_{23}\right)\right]>0, \\
\left(C_{11}+C_{22}-2 C_{12}\right)>0,\left(C_{11}+C_{33}-2 C_{13}\right)>0, \\
\left(C_{22}+C_{33}-2 C_{23}\right)>0,
\end{gathered}
$$

将表 2 数据代入可知, bc- $\mathrm{B}_{n} \mathrm{CN}(n=1,2,4)$ 的弹性常数 都满足该稳定性判据, 说明了这 3 种结构在力学上的 稳定性.

\section{2 弹性}

表 2 数据显示, 对于 bc-BCN, 有 $C_{33}>C_{11} \approx C_{22}$, 这表明 $\{001\}$ 平面内最邻近原子的结合强度最大, 而 $\{100\}$ 和 $\{010\}$ 平面内最邻近原子的结合强度较小, 并且这两者的结合强度相差不大; 在 $\mathrm{bc}-\mathrm{B}_{2} \mathrm{CN}$ 中, 有 $C_{33}>C_{11}>C_{22}$, 可知 $\{001\}$ 平面内最邻近原子的结合强 度最大, $\{010\}$ 的结合强度最小; 而在 bc- $\mathrm{B}_{4} \mathrm{CN}$ 中, $C_{33}>C_{22}>C_{11},\{001\}$ 的结合强度最大, $\{100\}$ 的结合强 度最小. 这 3 种材料都是在 $\{001\}$ 平面有最大的邻近 原子结合强度.

一般而言, 高硬度的材料具有较高的体弹模量, 但剪切模量与材料的硬度更具关联性. 表 3 列出了由 $\mathrm{LDA}$ 势场计算得到的 3 种 $\mathrm{bc}-\mathrm{B}_{n} \mathrm{CN}$ 结构的弹性模量、 泊松比以及 $G / B, B / C_{44}$ 和 $\Theta_{\mathrm{D}}$ 的值. 体弹模量 $B$ 、剪切 模量 $G$ 由 VRH 方法, 即公式(5)和(6)计算得到. 而 $E[100], E[010]$ 和 $E[001]$ 为单晶化合物在 [100], [010] 和[001]方向的杨氏模量, 它们可由 Castep 软件包直 接计算出来. 多晶体系下的杨氏模量 $E$ 和泊松比 $v$ 分 别为(11)和(12)式的计算结果, $\Theta_{\mathrm{D}}$ 的值则由公式 (13)-(16)计算得到. bc-BCN 结构具有最大的剪切模 量(主要来自 $C_{66}$ 和 $C_{13}$ 的贡献)和最大的 $G / B$. 共价键 材料, 如金刚石, 通常具有高剪切模量. 一般地, 共 价键材料的泊松比大约为 0.1 , 而金属材料的泊松比 大约为 $0.33^{[45]}$. 如果材料含有高度定向共价键, 则剪 切模量增加而泊松比会减小; 如果材料中的共价键 相对较短, 导致塑性变形过程中原子错位在该材料 中发生的可能性比具有非局域化长键材料的小很多. 如果包含太多非局域化键, 则它很可能是软材料. 由 文献[45]可以估计这 3 种结构的熔点分别为: bc-BCN
表 $3 \mathrm{bc}-\mathrm{B}_{n} \mathrm{CN}(n=1,2,4) 3$ 种结构模型的弹性模量 $E(\mathrm{GPa})$ 、 体弹模量 $(\mathrm{GPa})$ 、剪切模量 $G(\mathrm{GPa})$ 、泊松比 $v$ 和 $G / B$ 的值

Table 3 Theelastic modulus (GPa), bulk modulus (GPa), shear modulus (GPa), Poisson's ratio $v$ and Pugh modules ratio $G / B$ of bc- $\mathrm{B}_{n} \mathrm{CN}(n=1,2,4)$

\begin{tabular}{cccc}
\hline & $\mathrm{BCN}$ & $\mathrm{B}_{2} \mathrm{CN}$ & $\mathrm{B}_{4} \mathrm{CN}$ \\
\hline$E$ & 572 & 442 & 273 \\
$E[100]$ & 618 & 487 & 158 \\
$E[010]$ & 618 & 271 & 215 \\
$E[001]$ & 709 & 864 & 737 \\
$B$ & 298 & 264 & 225 \\
$G$ & 242 & 181 & 105 \\
$v$ & 0.181 & 0.221 & 0.298 \\
$G / B$ & 0.811 & 0.686 & 0.468 \\
$B / C_{44}$ & 1.355 & 1.859 & 1.875 \\
$\Theta_{\mathrm{D}}(\mathrm{K})$ & 1560 & 1383 & 1089 \\
\hline
\end{tabular}

$\left(5540^{\circ} \mathrm{C}\right), \mathrm{bc}-\mathrm{B}_{2} \mathrm{CN}\left(4610^{\circ} \mathrm{C}\right), \mathrm{bc}-\mathrm{B}_{4} \mathrm{CN}\left(3650^{\circ} \mathrm{C}\right)$. 与同 是共价键超硬材料金刚石的预测熔点 $9230^{\circ} \mathrm{C}$ 和 c-BN 的 $6390^{\circ} \mathrm{C}$ 相比, 本文 3 种材料的熔点已经低了很多, 表明在实验室合成时更容易实现.

bc-BCN, bc- $\mathrm{B}_{2} \mathrm{CN}$ 和 bc- $\mathrm{B}_{4} \mathrm{CN} 3$ 种晶体的泊松比 $v$ 的大小顺序为 $b c-B C N(0.181)<b c-B_{2} \mathrm{CN}(0.221)<b c-$ $\mathrm{B}_{4} \mathrm{CN}$ (0.298), 这说明材料 bc-BCN 的不可压缩性最 大. 体弹模量代表材料化合键强度的平均值, 而剪切 模量体现材料受到外力作用时抵抗化合键改变的能 力. 因此, $G / B$ 的大小反映材料中键合定向性的程度. 根据 Pugh 判据 ${ }^{[46]}, G / B>0.57$ 的材料整体显脆性; $G / B<0.57$ 的材料整体显韧性. 本文中, $G / B$ 值的顺 序是: bc- $\mathrm{BCN}(0.811)>b c-\mathrm{B}_{2} \mathrm{CN}(0.686)>0.57>b c-\mathrm{B}_{4} \mathrm{CN}$ (0.468), 表明 bc- $\mathrm{B}_{4} \mathrm{CN}$ 结构中化合键的定向性最低, 而 bc-BCN 结构中键的定向性最高, 并且 bc-BCN 和 bc- $B_{2} \mathrm{CN}$ 的 $G / B$ 都大于 0.57 , 因此它们都显脆性; 而 bc- $\mathrm{B}_{4} \mathrm{CN}$ 的 $G / B$ 值小于 0.57 , 显韧性. 泊松比反映了 材料在单轴形变情况下体积变化的大小, 当泊松比 等于 0.25 和 0.5 时分别代表中心力固体的上限和下限, 当等于 0.5 时表示弹性形变中体积不发生变化. 由表 3 所列的泊松比可以看出 bc-BCN 和 bc- $\mathrm{B}_{2} \mathrm{CN}$ 原子间 的结合力都不是中心力, bc- $\mathrm{B}_{4} \mathrm{CN}$ 的泊松比为 $0.25<0.298<0.5$, 其结合力属于中心力. 本文中 bc-BCN 和 bc- $\mathrm{B}_{2} \mathrm{CN}$ 的泊松比都小于 0.25 , 这说明形 变时体积将发生较大的变化; 而 bc- $\mathrm{B}_{4} \mathrm{CN}$ 相较而言 具有最大的泊松比, 形变时体积变化较小. 同时, 略 小的泊松比表明材料抵抗剪切应变的过程中具有较 好的稳定性. 


\section{3 硬度}

硬度代表了固体材料抵抗弹性变形、塑性变形或 者破坏的能力, 它是表征材料力学性能重要的物理 量. 材料硬度的计算可根据晶体原子间不同的键合 作用方式, 采用不同的公式进行推算. 对大多数陶瓷 或合金 ${ }^{[47,48]}$, 利用 Pugh 模量比(G/B)可较好地估计其 硬度. 而对于共价键晶体硬度的计算, 公式(22) ${ }^{[49]}$ 是 目前研究学者广泛采用 ${ }^{[19,50,51]}$ 且最为精准的计算方 法. 根据本文研究的晶体特性, 选用式(22)进行硬度 计算.

$$
H_{\mathrm{V}}=\left[\stackrel{x-y}{\Pi}\left(H_{\mathrm{V}}^{x-y}\right) n^{x-y}\right]^{1 / \Sigma \Sigma^{x-y}}
$$

式中

$$
\begin{gathered}
H_{\mathrm{V}}=350\left(N_{e}^{x-y}\right)^{2 / 3} \mathrm{e}^{-1.191 f_{i}^{x-y} /\left(d^{x-y}\right)^{2.5}}, \\
f_{i}^{1.36}=1-\mathrm{e}^{-\left|P_{\mathrm{c}}-P\right| / P},
\end{gathered}
$$

其中 $H_{\mathrm{V}}^{x-y}, n^{x-y}, d^{x-y}, f_{i}^{x-y}$ 和 $P$ 分别代表晶体由纯 $x-y$ 型键搭建时的硬度、键数、键长、Phillips 离子性 和键布居数. 本文中的 $P_{\mathrm{c}}=0.75, N_{\mathrm{e}}^{x-y}$ 为单位 $\AA^{3}$ 中 $x-y$ 型键中价电子的数目. bc- $\mathrm{B}_{n} \mathrm{CN}(n=1,2,4)$ 的硬度、键 的个数、各原子间电子云重叠布居以及各原子键长列 于表 4 中.

由表 4 的计算结果可得, 本文所研究的 3 种化合 物都是超硬材料. 由于 $b c-B C N, b c-B_{2} C N$ 和 $b c-B_{4} C N$ 的硬度逐渐减小, 表明硬度随着 $\mathrm{B}$ 原子比例的增多 而变小. 观察每种结构中所含键的种类我们不难发 现, bc-BCN 结构中不含 B-B 键, 而且每个晶胞含有 相同数量的 $\mathrm{B}-\mathrm{N}, \mathrm{B}-\mathrm{C}, \mathrm{C}-\mathrm{N}$ 键; bc- $\mathrm{B}_{2} \mathrm{CN}$ 中 $\mathrm{C}-\mathrm{N}$ 和 $\mathrm{B}-\mathrm{B}$ 键较少, 具有和 $\mathrm{bc}-\mathrm{BCN}$ 结构数量相同的 $\mathrm{B}-\mathrm{N}$ 和 $\mathrm{B}-\mathrm{C}$ 键; 而 $\mathrm{bc}-\mathrm{B}_{4} \mathrm{CN}$ 中的 $\mathrm{B}-\mathrm{B}$ 键含量最多, $\mathrm{B}-\mathrm{N}$ 和 $\mathrm{B}-\mathrm{C}$ 两种键的数目都少于 $b c-B C N$ 和 bc- $\mathrm{B}_{2} \mathrm{CN}$. 键的种类和数目也决定了材料硬度的高低, 这解释了本文中 bc-BCN $(61 \mathrm{GPa})>b c-\mathrm{B}_{2} \mathrm{CN}(56 \mathrm{GPa})>$ $b c-B_{4} \mathrm{CN}(48 \mathrm{GPa})$ 的原因. 由于 $\mathrm{B}-\mathrm{B}$ 键的硬度较小, $\mathrm{B}-\mathrm{N}, \mathrm{B}-\mathrm{C}, \mathrm{C}-\mathrm{N}$ 键硬度相对较高, 因此当 $\mathrm{B}-\mathrm{B}$ 键 的比例增加, 而 $\mathrm{B}-\mathrm{N}, \mathrm{B}-\mathrm{C}, \mathrm{C}-\mathrm{N}$ 键的数量减少时, 就会出现硬度降低的情况. 表 4 中在每种模型硬度的 右方给出了对应的硬弹比(硬度比弹性模量), 它的大 小表征材料的耐磨性. 数据表明, bc-BCN, bc- $\mathrm{B}_{2} \mathrm{CN}$ 和 $b c-\mathrm{B}_{4} \mathrm{CN}$ 的耐磨性逐渐增大. 尽管 $\mathrm{bc}-\mathrm{B}_{4} \mathrm{CN}$ 的硬度 在三者中最小, 但其耐磨性最好.

\section{4 各向异性}

\subsection{1 弹性各向异性}

当研究材料的力学性质时, 我们通常会考虑微 裂纹和晶格畸变对材料力学性能的影响, 而弹性性 质的各向异性通常是微裂纹和晶格畸变产生的决定 因素. 对超硬材料而言, 其各向异性越小更利于实际 应用. 因而对其弹性性质的各向异性研究有重要意 义. 为了定量研究单晶的各向异性, Ranganathan 等 人 ${ }^{[52]}$ 引入了适用于所有晶相的普适弹性各向异性指 数 $A^{\mathrm{U}}$

$$
A^{\mathrm{U}}=5 \frac{G_{\mathrm{V}}}{G_{\mathrm{R}}}+\frac{B_{\mathrm{v}}}{B_{\mathrm{R}}}-6 .
$$

bc-BCN, bc- $\mathrm{B}_{2} \mathrm{CN}$ 和 bc- $\mathrm{B}_{4} \mathrm{CN} 3$ 种结构模型的各 向异性指数如表 5 所示. 表 5 中同时列出了金刚石和 立方氮化硼的数据, 以便对比. $A^{\mathrm{U}}=0$ 即说明单晶呈各 向同性, $A^{\mathrm{U}}$ 的值对 0 的偏离越大, 材料的各向异性程 度也越大. 表 5 中所示 bc- $\mathrm{BCN}, \mathrm{bc}-\mathrm{B}_{2} \mathrm{CN}$ 和 $b c-\mathrm{B}_{4} \mathrm{CN}$ 的 $A^{\mathrm{U}}$ 的值分别为 $0.1114,0.948,2.551$ 均大于 0 , 说明 这 3 种材料弹性都呈现各向异性. 且 $A^{\mathrm{U}}$ 的值以 bc-BCN, bc- $\mathrm{B}_{2} \mathrm{CN}, \mathrm{bc}-\mathrm{B}_{4} \mathrm{CN}$ 的顺序依次增大, 因此, 其各向异性程度随 $\mathrm{B}$ 原子的增加而增大.

另外, Chung 和 Buessem ${ }^{[53]}$ 提出了各向异性分数 比的概念, 其表达式如下:

$$
\begin{aligned}
& A_{\mathrm{G}}=\frac{G_{\mathrm{V}}-G_{\mathrm{R}}}{G_{\mathrm{V}}+G_{\mathrm{R}}}, \\
& A_{\mathrm{B}}=\frac{B_{\mathrm{V}}-B_{\mathrm{R}}}{B_{\mathrm{V}}+B_{\mathrm{R}}} .
\end{aligned}
$$

$A_{\mathrm{G}}$ 和 $A_{\mathrm{B}}$ 通常用来判断材料剪切模量和体弹模量 的各向异性程度. 当 $A_{\mathrm{G}}$ 和 $A_{\mathrm{B}}$ 的值为 0 时, 材料的性 质表现为各向同性; 而当 $A_{\mathrm{G}}$ 和 $A_{\mathrm{B}}$ 的值为 1 时, 材料 的各向异性程度最大. 从表 5 中可看出 3 种 $\mathrm{bc}-\mathrm{B}_{n} \mathrm{CN}$ 结构 $A_{\mathrm{G}}$ 和 $A_{\mathrm{B}}$ 的值都不为 0 , 说明它们都表现为各向 异性, 而且它们的值以 $n=4,2,1$ 的顺序逐渐减小, 所 以剪切模量和体弹模量的各向异性程度也随之减小. 这与上述分析的普适弹性各向异性指数 $A^{\mathrm{U}}$ 的变化一 致. 另外, $A_{\mathrm{G}}$ 和 $A_{\mathrm{B}}$ 分别被用于判断材料的剪切模量 和体弹模量的各向异性程度, 它们的值都不为零, 表 明 bc- $\mathrm{BCN}, \mathrm{bc}-\mathrm{B}_{2} \mathrm{CN}$ 和 bc- $\mathrm{B}_{4} \mathrm{CN}$ 的剪切模量和体弹 模量的各向异性程度也以 $n=4,2,1$ 的顺序逐渐减小.

为了定量地研究单晶的各向异性, 我们计算了 描述单晶弹性各项异性因子 Zener 因子 ${ }^{[30]}$ 
胡萌等. 中国科学: 物理学 力学 天文学 2016 年 第 46 卷 第 6 期

表 $4 \mathrm{bc}-\mathrm{B}_{n} \mathrm{CN}(n=1,2,4)$ 键的个数 $n_{\mathrm{i}}$ 、各原子间电子云重叠布居 $P^{x-y}$ 及各原子键长 $d^{x-y}(\AA)$

Table 4 Bond type, bond number $n_{i}$, bond population $P^{x-y}$ and bond length $d^{x-y}(\AA)$ of bc- $\mathrm{B}_{n} \mathrm{CN}(n=1,2,4)$

\begin{tabular}{|c|c|c|c|c|c|c|c|c|c|c|c|c|c|c|}
\hline & \multirow{2}{*}{ Hardness } & \multirow{2}{*}{$H_{\mathrm{v}} / E$} & \multicolumn{3}{|c|}{$\mathrm{B}-\mathrm{N}$ bond } & \multicolumn{3}{|c|}{$\mathrm{B}-\mathrm{C}$ bond } & \multicolumn{3}{|c|}{$\mathrm{C}-\mathrm{N}$ bond } & \multicolumn{3}{|c|}{ B-B bond } \\
\hline & & & $n_{\mathrm{i}}$ & $P^{\mathrm{B}-\mathrm{N}}$ & $d^{\mathrm{B}-\mathrm{N}}$ & $n_{\mathrm{i}}$ & $P^{\mathrm{B}-\mathrm{N}}$ & $d^{\mathrm{B}-\mathrm{N}}$ & $n_{\mathrm{i}}$ & $P^{\mathrm{B}-\mathrm{N}}$ & $d^{\mathrm{B}-\mathrm{N}}$ & $n_{\mathrm{i}}$ & $P^{\mathrm{B}-\mathrm{N}}$ & $d^{\mathrm{B}-\mathrm{N}}$ \\
\hline \multirow{2}{*}{$\mathrm{BCN}$} & \multirow{2}{*}{61} & \multirow{2}{*}{0.11} & 4 & \multirow{2}{*}{0.55} & 1.59760 & 4 & \multirow{2}{*}{0.80} & 1.54711 & 4 & \multirow{2}{*}{0.72} & 1.50074 & - & - & - \\
\hline & & & 4 & & 1.59769 & 4 & & 1.54720 & 4 & & 1.50077 & - & - & - \\
\hline \multirow{4}{*}{$\mathrm{B}_{2} \mathrm{CN}$} & \multirow{4}{*}{56} & \multirow{4}{*}{0.13} & 2 & 0.68 & 1.49478 & 2 & 0.86 & 1.51064 & 2 & 0.68 & 1.51088 & 4 & 0.59 & 1.70792 \\
\hline & & & 2 & 0.66 & 1.51401 & 2 & 0.80 & 1.54853 & 2 & 0.70 & 1.51454 & - & - & - \\
\hline & & & 4 & 0.62 & 1.59566 & 2 & 0.79 & 1.61878 & - & - & - & - & - & - \\
\hline & & & - & - & - & 2 & 0.74 & 1.61814 & - & - & - & - & - & - \\
\hline \multirow{4}{*}{$\mathrm{B}_{4} \mathrm{CN}$} & \multirow{4}{*}{48} & \multirow{4}{*}{0.18} & 2 & 0.74 & 1.46526 & 2 & 0.90 & 1.59794 & 2 & 0.68 & 1.50249 & 2 & 0.79 & 1.64618 \\
\hline & & & 2 & 0.67 & 1.49828 & 4 & 0.69 & 1.60789 & - & - & - & 2 & 0.65 & 1.68099 \\
\hline & & & 2 & 0.68 & 1.55479 & - & - & - & - & - & - & 4 & 0.57 & 1.75513 \\
\hline & & & - & - & - & - & - & - & - & - & - & 2 & 0.58 & 1.80687 \\
\hline
\end{tabular}

表 5 普适弹性各向异性指数 $A^{\mathrm{U}}$ 和各向异性分数比 $A_{\mathrm{B}}, A_{\mathrm{G}}$ 以及剪切各向异性因子 $A_{1}, A_{2}$ 和 $A_{3}$

Table 5 Universal elastic anisotropic index $A^{\mathrm{U}}$, percent elastic anisotropy $A_{\mathrm{B}}$ and $A_{\mathrm{G}}$, shear anisotropic factors $A_{1}, A_{2}$ and $A_{3}$ of bc$\mathrm{B}_{n} \mathrm{CN}(n=1,2,4)$, Diamond and c-BN

\begin{tabular}{cccccc}
\hline & $\mathrm{BCN}$ & $\mathrm{B}_{2} \mathrm{CN}$ & $\mathrm{B}_{4} \mathrm{CN}$ & Diamond & $\mathrm{c}-\mathrm{BN}$ \\
\hline$A^{\mathrm{U}}$ & 0.1114 & 0.948 & 2.551 & 0.0549 & 0.1912 \\
$A_{\mathrm{G}}$ & 0.0110 & 0.077 & 0.195 & 0.0055 & 0.0188 \\
$A_{\mathrm{B}}$ & 0.0001 & 0.056 & 0.062 & 0 & 0 \\
$A_{1}$ & 0.7325 & 0.435 & 0.508 & 1.2380 & 1.4867 \\
$A_{2}$ & 0.7322 & 0.738 & 0.219 & 1.2380 & 1.4867 \\
$A_{3}$ & 0.8193 & 1.092 & 1.793 & 1.2380 & 1.4867 \\
\hline
\end{tabular}

$$
\begin{aligned}
& A_{1}=\frac{4 C_{44}}{C_{11}+C_{33}-2 C_{13}}, \\
& A_{2}=\frac{4 C_{55}}{C_{22}+C_{33}-2 C_{23}}, \\
& A_{3}=\frac{4 C_{66}}{C_{11}+C_{22}-2 C_{12}},
\end{aligned}
$$

式中 $C_{i j}$ 是弹性常数. $A_{1}$ 表示 $\{100\}$ 剪切面沿 $<011>$ 和 晶向 $<010>$ 的剪切模量之比, $A_{2}$ 表示 $\{010\}$ 剪切面沿 $<101>$ 和晶向 $<001>$ 的剪切模量之比, $A_{3}$ 表示的是 $\{001\}$ 剪切面沿 $<110>$ 和晶向 $<010>$ 的剪切模量之比. Zener 因子的值都为 1 时说明晶体弹性性质呈各向同 性，当其中有一个不为 1 时说明这种晶体是各向异性 的. 表 5 中给出的 $A_{1}, A_{2}$ 和 $A_{3}$ 的值都不为 1 , 说明这 3 种晶体都是各向异性的. bc-BCN, bc- $\mathrm{B}_{2} \mathrm{CN}$ 和 bc- $\mathrm{B}_{4} \mathrm{CN}$ 这 3 种晶体在 $\{100\},\{010\}$ 和 $\{001\}$ 三个面的 剪切各向异性由小到大的顺序为 bc-BCN: $\{001\}$, $\{100\},\{010\}, \quad b c-B_{2} C N:\{001\},\{010\},\{100\}$, bc- $\mathrm{B}_{4} \mathrm{CN}:\{100\},\{001\},\{010\}$, 而且 bc-BCN 中的 $\{100\}$ 和 $\{010\}$ 两个平面的各向异性程度相差不大,
甚至可以说各向异性程度相同. 与 Diamond 对比发 现 (见表 5), bc- $\mathrm{B}_{n} \mathrm{CN}$ 这 3 种材料的普适弹性各向异性 指数 $A^{\mathrm{U}}$ 、分数比各向异性 $A_{\mathrm{B}} 、 A_{\mathrm{G}}$ 的值都比 Diamond 大, 说明 $\mathrm{bc}-\mathrm{B}_{n} \mathrm{CN}(n=1,2,4)$ 的各向异性比 Diamond 严重; 与 $\mathrm{c}-\mathrm{BN}$ 的各向异性判断参数对比(见表 5), bc-BCN 的各向异性要比 c-BN 小, 而 bc- $\mathrm{B}_{2} \mathrm{CN}$ 和 $\mathrm{bc}-\mathrm{B}_{4} \mathrm{CN}$ 的各向异性都大于 $\mathrm{c}-\mathrm{BN}$, 表明了 bc-BCN 比 $\mathrm{c}-\mathrm{BN}$ 的各向异性程度小, 因此 bc-BCN 相对于 c-BN 在变形过程中更不容易形成微裂纹, 更适合在有外 应力环境中的应用, 而 bc- $\mathrm{B}_{2} \mathrm{CN}$ 和 bc- $\mathrm{B}_{4} \mathrm{CN}$ 在某些方 向上的各向异性程度甚至小于 Diamond, 值得在各向 异性的应用上进一步研究.

为了能更直观地体现 bc- $\mathrm{B}_{n} \mathrm{CN}(n=1,2,4)$ 的弹性 各向异性, 图 2 给出了 bc-BCN, bc- $\mathrm{B}_{2} \mathrm{CN}$ 和 bc- $\mathrm{B}_{4} \mathrm{CN}$ 的杨氏模量在空间中的三维分布. 对于杨氏模量, 由 于其应力与受力面具有相同的方向, 因此可以借助 图形直观地判断不同方向承受外加应力的能力. 三 维立体杨氏模量的计算公式 ${ }^{[54]}$ 如下:

$$
\frac{1}{E}=S_{1111}^{\prime}=\sum_{n=1}^{3} \sum_{m=1}^{3} \sum_{p=1}^{3} \sum_{q=1}^{3} S_{n m p q} l_{1 n} l_{1 m} l_{1 p} l_{1 q},
$$

式中 $S_{n m p q}$ 为弹性顺度系数 $\left(S_{n m p q}=C_{i j k d}^{-1}\right), l_{1 n}, l_{1 m}$, $l_{1 p}, l_{1 q}$ 代表方向余弦. 图像与标准球形的偏差程度代 表了其各向异性的程度.

从图中我们可以看出杨氏模量的三维立体图都 呈不规则的球状, 表现为各向异性. 图 2 中, bc-BCN 在 [100], [010], [001] 三个方向上的杨氏模量较其他高 指数晶向更大; $b c-B_{2} \mathrm{CN}$ 结构在 [110]和 [1 10$]$ 方向上 的杨氏模量形状都呈凸起状, 在 [010]方向上也有外 凸的趋势, 但[100]方向上的杨氏模量图却呈现略 

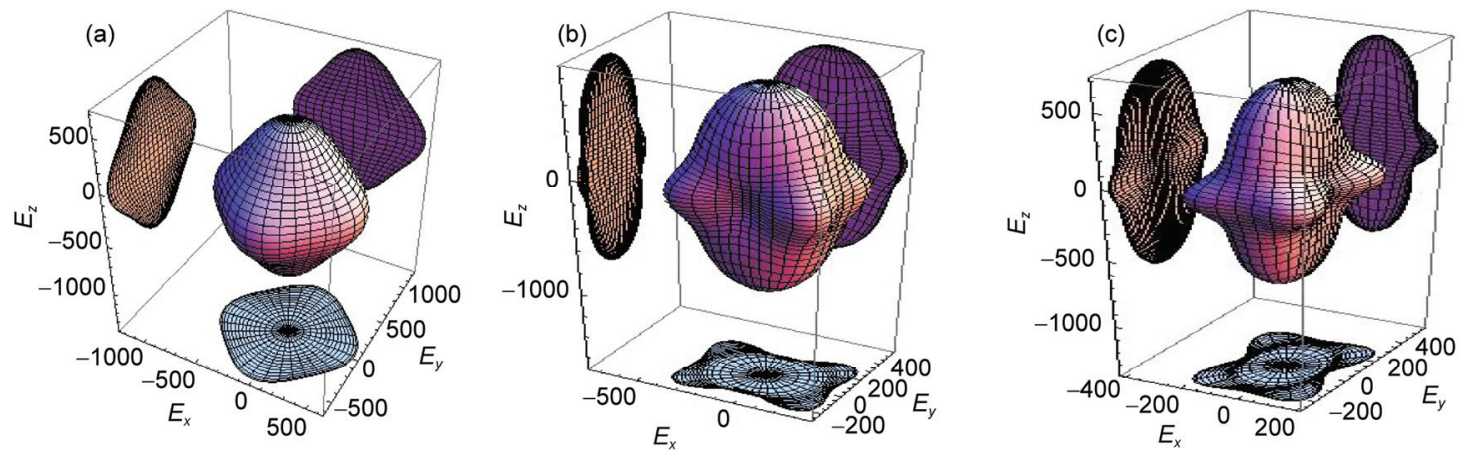

图 2 (网络版彩图) 杨氏模量的三维立体图 (a) bc-BCN; (b) bc- $\mathrm{B}_{2} \mathrm{CN}$; (c) bc- $\mathrm{B}_{4} \mathrm{CN}$

Figure 2 (Color online) Three-dimensional stereo grams of the Young's modulus (GPa) of bc- $\mathrm{B}_{n} \mathrm{CN}\left(n=1,2\right.$, 4). (a) bc-BCN; (b) bc- $\mathrm{B}_{2} \mathrm{CN}$; (c) bc- $\mathrm{B}_{4} \mathrm{CN}$.

微向内凹陷的状态, 并且在[001]方向上具有最大的 杨氏模量值，表现为沿 [001]方向最难发生形变;

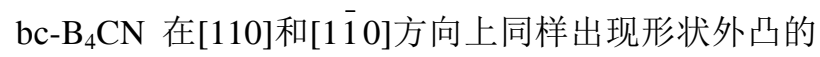
现象，但 [010]和 [100]方向上的图形凹凸情况却与 bc- $\mathrm{B}_{2} \mathrm{CN}$ 结构刚好相反, 并且 bc- $\mathrm{B}_{4} \mathrm{CN}$ 结构的杨氏模 量在 $(001)$ 平面的投影显示, bc- $\mathrm{B}_{4} \mathrm{CN}$ 的投影图形状和 bc- $\mathrm{B}_{2} \mathrm{CN}$ 在此平面上的投影图旋转 $90^{\circ}$ 的形状类似, 表明 bc- $\mathrm{B}_{2} \mathrm{CN}$ 和 bc- $\mathrm{B}_{4} \mathrm{CN}$ 在[100]和[010]方向承受外 加应力产生形变的方向几乎正交. 结合表 3 可知, $b c-B C N, b c-B_{2} C N$ 和 $b c-B_{4} C N$ 在 [001]方向上的杨氏 模量都是最大的, 而且杨氏模量最大值和最小值相 差最大的是 bc- $\mathrm{B}_{4} \mathrm{CN}$. 由此可知, $\mathrm{B}$ 原子比例的增加, 改变了 $\mathrm{bc}-\mathrm{B}_{n} \mathrm{CN}$ 结构中键的种类以及分布情况, 最 终导致了弹性模量的各向异性有不同程度的增大.

综上所述, 3 种材料中 bc-BCN 的各向异性最弱, 且小于 c-BN. 如能实现合成, 其工业应用将比 c-BN 更广.

\subsection{2 应力应变曲线及塑性各向异性}

为了分析 $\mathrm{bc}-\mathrm{B}_{n} \mathrm{CN}$ 的力学形变机制, 我们通过 对原胞在不同方向上的拉伸和剪切, 得到理想应力应变曲线. 如图 3 通过逐步增加应变逐步弛豫的方法, 计算得到材料在不同应变下的应力, 这种应力变化 所体现的是材料从弹性区域变到不稳定区域直至最 终崩溃的过程. 而曲线的转折点是由于施加到一定 程度的应变时, 原子挣脱近邻原子的束缚, 自发地从 初始的晶格点阵位置滑移到一个新的点阵位置上以 达到能量最低状态的原子变化引起的.

从图 3 可以看出当对 bc- $\mathrm{BCN}, \mathrm{bc}-\mathrm{B}_{2} \mathrm{CN}, \mathrm{bc}-\mathrm{B}_{4} \mathrm{CN}$ 施加拉伸形变时, 晶体的应力应变曲线呈现出明显
的各向异性. 这是由于晶胞在沿不同晶向拉伸时参 与形变的键不同, 以及形变过程中应力与键合初始 方向的夹角不同导致的. 刚开始施加形变时, 应力和 应变成线性变化. bc-BCN 结构拉伸时在 [100]和[010] 方向上曲线是重合的，其他方向上的曲线则没有出 现该现象, 这与图 2 所呈现的 3 种结构的杨氏模量各 向异性特征一致. 从图 3 我们可以看出, 在 [100]晶向 上, bc-BCN 和 bc- $\mathrm{B}_{2} \mathrm{CN}$ 的有效区域是应变都在 $20 \%$ 以内, 其中拉伸强度最大的是 bc-BCN, 强度为 76.1 $\mathrm{GPa}, \mathrm{bc}-\mathrm{B}_{4} \mathrm{CN}$ 在该方向上的应力-应变曲线一直呈现 上升趋势, 说明其在 [100]方向没有出现理想应力, 并且 $b c-\mathrm{B}_{4} \mathrm{CN}$ 的应力应变曲线一直处于 $b c-B C N$ 和 $b c-B_{2} C N$ 曲线的下方, 这也是由于 $\mathrm{B}-\mathrm{B}$ 键能量最低 而在 $\mathrm{bc}-\mathrm{B}_{4} \mathrm{CN}$ 结构中含有最多的 $\mathrm{B}-\mathrm{B}$ 键所致. 在 [010]晶向上, 拉伸强度最大的仍然是 bc-BCN, 强度 为 $76.1 \mathrm{GPa}$, 而 $\mathrm{bc}-\mathrm{B}_{2} \mathrm{CN}$ 在[010]方向上应力应变曲 线一直呈现上升趋势. 在 [001]晶向上, bc- $\mathrm{B}_{2} \mathrm{CN}$ 的应 力应变曲线的有效区域相对较短, 应变达到 $16 \%$ 后 就进入断裂阶段, 具有最大的应力 $72.3 \mathrm{GPa}$, 而且 $b c-B C N, b c-B_{2} C N$ 和 $b c-B_{4} C N$ 在[001]晶向上都具有 最大的理想强度, 与杨氏模量的三维立体图(图 2)分 析得到的结果一致. 这 3 种结构拉伸强度的各向异性 程度为 bc-BCN: $\sigma_{[100]}(76 \mathrm{GPa}): \sigma_{[010]}(76 \mathrm{GPa}): \sigma_{[001]}(107$ $\mathrm{GPa}) \approx 1: 1: 1.41 ; \mathrm{bc}-\mathrm{B}_{2} \mathrm{CN}: \sigma_{[100]}(70 \mathrm{GPa}): \sigma_{[010]}(58 \mathrm{GPa}):$ $\sigma_{[001]}(106 \mathrm{GPa}) \approx 1: 0.83: 1.51 ; \mathrm{bc}-\mathrm{B}_{4} \mathrm{CN}: \sigma_{[100]}(44 \mathrm{GPa}):$ $\sigma_{[010]}(65 \mathrm{GPa}): \sigma_{[001]}(72 \mathrm{GPa}) \approx 1: 1.49: 1.65$. 由此可知 bc- $\mathrm{BCN}, \mathrm{bc}-\mathrm{B}_{2} \mathrm{CN}, \mathrm{bc}-\mathrm{B}_{4} \mathrm{CN}$ 在[001]方向上拉伸的各 向异性最大, 并且各向异性的程度随着 $\mathrm{B}$ 原子比例 的增加而增大.

对于剪切曲线的线性部分(应变小于 $2 \%$ 时), 我 


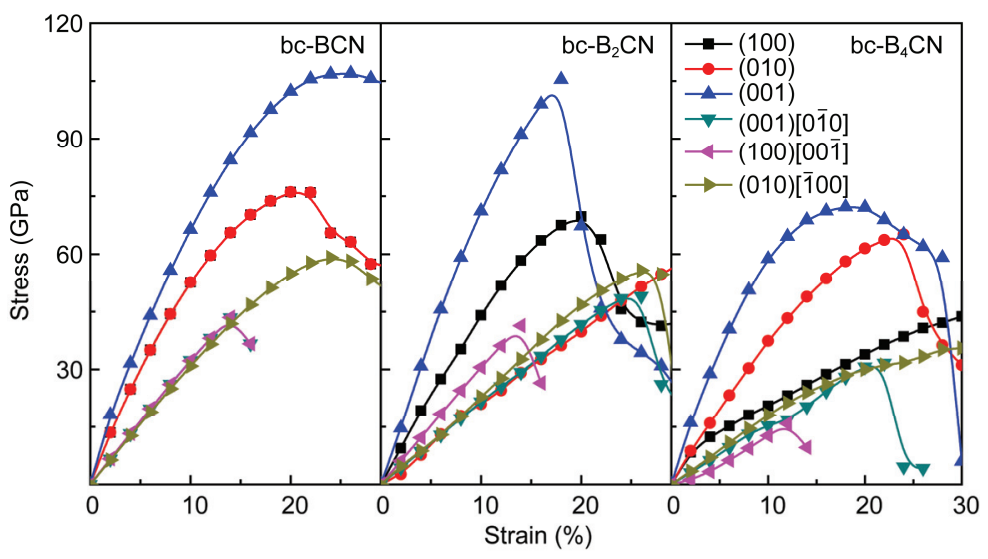

图 3 (网络版彩图) bc- $\mathrm{BCN}, \mathrm{bc}-\mathrm{B}_{2} \mathrm{CN}$ 和 $\mathrm{bc}-\mathrm{B}_{4} \mathrm{CN}$ 在不同方向上的拉伸以及剪切应力-应变曲线

Figure 3 (Color online) The tensile and shear stress-strain curves of bc- $\mathrm{B}_{n} \mathrm{CN}(n=1,2,4)$ along different directions.

们可以推测出这 3 种晶体在不同方向上的剪切模量: bc-BCN $\left(\mathrm{G}_{(100)[0 \overline{0} 0]}=330 \mathrm{GPa}, \mathrm{G}_{(010)[00 \overline{1}]}=332 \mathrm{GPa}\right.$, $\left.\mathrm{G}_{(001)[\overline{1} \mathbf{0}]}=318 \mathrm{GPa}\right)$, bc- $\mathrm{B}_{2} \mathrm{CN} \quad\left(\mathrm{G}_{(100)[0 \overline{1} \mathrm{O}]}=216 \mathrm{GPa}\right.$, $\left.\mathrm{G}_{(010)[00 \overline{1}]}=305 \mathrm{GPa}, \mathrm{G}_{(100)[0 \overline{10}]}=238 \mathrm{GPa}\right)$, bc- $\mathrm{B}_{4} \mathrm{CN}$ $\left(\mathrm{G}_{(100)[0 \overline{1} 0]}=154 \mathrm{GPa}, \mathrm{G}_{(010)[00 \overline{1}]}=61 \mathrm{GPa}, \mathrm{G}_{(100)[0 \overline{1} \mathrm{0}]}=\right.$ $174 \mathrm{GPa}) . C_{44}$ 表示晶格对于(100)面上沿[010]晶向剪 切应变的抵抗能力, $C_{55}$ 表示(010)剪切面沿[001] 晶 向对剪切应变的抵抗能力, $C_{66}$ 则表示(001)剪切面沿 [100]晶向剪切应变的抵抗能力. 如表 2 所示, bc-BCN: $\left.C_{44}=220 \mathrm{GPa}, C_{55}=220 \mathrm{GPa}, C_{66}=211 \mathrm{GPa}\right), \mathrm{bc}-\mathrm{B}_{2} \mathrm{CN}$ : $\left.C_{44}=142 \mathrm{GPa}, C_{55}=195 \mathrm{GPa}, C_{66}=151 \mathrm{GPa}\right), \mathrm{bc}-\mathrm{B}_{4} \mathrm{CN}$ : $\left.C_{44}=120 \mathrm{GPa}, C_{55}=50 \mathrm{GPa}, C_{66}=107 \mathrm{GPa}\right)$, 对比发现 计算结果与上述推测所得剪切模量大小的规律相符 合, 这主要是由于剪切时抵抗不同种类键的形变能 力不同所导致. 由表 3 可知剪切模量的理论值分别为 bc-BCN(238 GPa), bc- $\mathrm{B}_{2} \mathrm{CN}(161 \mathrm{GPa}), \mathrm{bc}-\mathrm{B}_{4} \mathrm{CN}(114$ $\mathrm{GPa})$, 这进一步表明了剪切模量是各项异性的, 与表 5 中 $A_{\mathrm{G}}$ 显示出的各向异性规律一致. 剪切时, 随着剪 切应变的增大, 在应力达到最大值前的线性变化区 间内, 3 种结构在(001) [0 $\overline{1} 0]$, (100) [00 $\overline{1}],(010)$ [ $\overline{1} 00]$ 方向上的应力变化趋势都是一致的, 其中上升最快 的都是 bc-BCN, 斜率最小的都为 $b c-\mathrm{B}_{4} \mathrm{CN}$, 而且 bc-BCN 最先达到解离状态. 在 (100) [0 $\overline{1} 0]$ 方向上 $\mathrm{bc}-\mathrm{BCN}$ 和 $\mathrm{bc}-\mathrm{B}_{4} \mathrm{CN}$ 的应力-应变曲线崩溃较快, 说明 $b c-B C N$ 和 $b c-B_{4} C N$ 在这一方向上不如其他两个方向 稳定, 而 $\mathrm{bc}-\mathrm{B}_{2} \mathrm{CN}$ 在这三个方向上都是在应变达到 $24 \%$ 才进入断裂阶段. 剪切时 bc-BCN, bc- $\mathrm{B}_{2} \mathrm{CN}$,
$\mathrm{bc}-\mathrm{B}_{4} \mathrm{CN}$ 在三个方向上的最大剪切强度中的最小的 强度分别为: $b c-B C N\left(G_{(100)[0 \overline{1} 0]}=44 \mathrm{GPa}\right), b c-\mathrm{B}_{2} \mathrm{CN}$ $\left(\mathrm{G}_{(100)[0 \overline{0} \overline{0}]}=41 \mathrm{GPa}\right), \mathrm{bc}-\mathrm{B}_{4} \mathrm{CN}\left(\mathrm{G}_{(100)[0 \overline{\mathrm{1}} \mathrm{O}]}=16 \mathrm{GPa}\right)$, 它 们都在计算所得的硬度范围内 bc-BCN(62 GPa), $b c-B_{2} \mathrm{CN}(56 \mathrm{GPa}), b c-\mathrm{B}_{4} \mathrm{CN}(46 \mathrm{GPa})$. 另外, bc-BCN 的应力应变曲线呈现出一个独特的特点, (001) [ $\overline{1} 00]$ 和(100) [0 $\overline{1} 0]$ 的剪切曲线形状基本上是完全重合的,

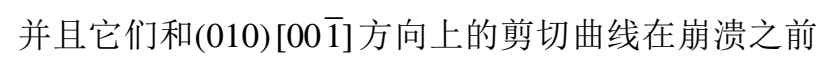
的形状也大体上一致, 这起因于 $\mathrm{bc}-\mathrm{BCN}$ 具有较弱的 各向异性. 由表 2 可知, bc-BCN 结构中的 $C_{44} / C_{66}$ 和 $C_{55} / C_{66}$ 的值都接近 1 , 这表明它的剪切刚度是近似各 项同性的; 相反地, 对于 bc- $\mathrm{B}_{2} \mathrm{CN}$ 和 bc- $\mathrm{B}_{4} \mathrm{CN}$ 这两种 结构而言, 它们的剪切刚度则是各向异性的, 而且 bc- $\mathrm{B}_{4} \mathrm{CN}$ 的 $C_{44} / C_{66}$ 和 $C_{55} / C_{66}$ 的值较 bc- $\mathrm{B}_{2} \mathrm{CN}$ 而言偏 离 1 较多, 因此它们的剪切刚度也随着 $\mathrm{B}$ 原子含量的 增加而增大.

\section{4 结论}

我们采用基于密度泛函理论的第一性原理方法 计算分析了 3 种 $\mathrm{bc}-\mathrm{B}_{n} \mathrm{CN}(n=1,2,4)$ 晶体的弹性、硬 度及各向异性. 本文所研究的 3 种结构中, bc-BCN 具 有绝对值最大的形成焓和结合能, 决定了 bc-BCN 超 硬材料的最易合成性和最佳的稳定性. 基于键能和 键硬度计算分析得到, $\mathrm{B}-\mathrm{B}$ 键相对于 $\mathrm{B}-\mathrm{N}, \mathrm{B}-\mathrm{C}$, $\mathrm{C}-\mathrm{N}$ 键具有较弱的结合强度, 从而指出 bc-BCN 结 构的相对稳定性起因于其含有较多较强作用的 B- $\mathrm{N}$, 
$\mathrm{B}-\mathrm{C}, \mathrm{C}-\mathrm{N}$ 键, 而不含弱作用的 $\mathrm{B}-\mathrm{B}$ 键. $\mathrm{bc}-\mathrm{B}_{n} \mathrm{CN}$ $(n=1,2,4)$ 的弹性模量、硬度的计算结果与熔点呈现 出一致的变化规律: bc-BCN $>b c-B_{2} C N>b c-B_{4} C N$. 静 态和动态的弹性性能及其各向异性计算分析表明,
bc-BCN 在 3 种结构中呈现出最大的硬度和弹性模量, 并具有最优的弹性各向异性和塑性各向异性. bc-BCN 结构的易形成性和优良的力学综合性能决定 了它具有更宽广的应用前景.

\section{参考文献}

1 Sun J, Wang H T, Ming N, et al. Optical properties of heterodiamond $\mathrm{B}_{2} \mathrm{CN}$ using first-principles calculations. Appl Phys Lett, 2004, 84: 4544-4546

2 Zhang Y, Sun H, Chen C F. Superhard cubic $\mathrm{BC}_{2} \mathrm{~N}$ compared to diamond. Phys Rev Lett, 2004, 93: 195504

3 Zhao Y, He D W, Daemen L L, et al. Superhard B-C-N materials synthesized in nanostructured bulks. J Mater Res, 2002, 17: 3139-3145

4 Zhou X F, Sun J, Fan Y X, et al. Most likely phase of superhard $\mathrm{BC}_{2} \mathrm{~N}$ by ab initio calculations. Phys Rev B, 2007, 76: 100101

5 Tateyama Y, Ogitsu T, Kusakabe K, et al. Proposed synthesis path for heterodiamond $B_{2}$ N. Phys Rev B, 1997, 55: R10161

6 Ray S C, Tsai H M, Bao C W, et al. Electronic and bonding structures of BCN thin films investigated by $\mathrm{X}$ ray absorption and photoemission. J Appl Phys, 2004, 96: 208-211

7 Kim E, Pang T, Utsumi W, et al. Cubic phases of $\mathrm{BC}_{2} \mathrm{~N}$ : A first-principles study. Phys Rev B, 2007, 75: 184115

8 Luo X G, Guo X J, Liu Z Y, et al. First-principles study of wurtzite BC $_{2}$ N. Phys Rev B, 2007, 76: 092107

9 He J L, Guo L C, Wu E, et al. First-principles study of $\mathrm{B}_{2} \mathrm{CN}$ crystals deduced from the diamond structure. J Phys Condens Mater, 2004, 16: $8131-8138$

10 Vel L, Demazeau G, Etourneau J. Cubic boron-nitride-synthesis, physicochemical properties and applications. Mater Sci Eng B, 1991, 10: $149-164$

11 Singh B P. Characterization of cubic boron-nitride comoacts. Mater Res Bull, 1986, 21: 85-92

12 Andrievski R A. Superhard materials based on nanostructured high-melting point compounds: Achievements and perspectives. Int J Refract Met H, 2001, 19: 447-452

13 Badzian A R. Cubic boron-nitride-diamond mixed-crystrals. Mater Res Bull, 1981, 16: 1385-1393

14 Solozhenko V L, Andrault D, Fiquet G, et al. Synthesis of superhard cubic BC 2 N. Appl Phys Lett, 2001, 78: 1385-1387

15 Sasaki T, Akaishi M, Yamaoka S, et al. Simultaneous crystallization of diamond and cubic boron nitride from the graphite relative boron carbide nitride $\left(\mathrm{BC}_{2} \mathrm{~N}\right)$ under high pressure/high temperature conditions. Chem Mater, 1993, 5: 695-699

16 Yao B, Liu L, Su W H. Formation, characterization, and properties of a new boron-carbon-nitrogen crystal. J Appl Phys, 1999, 86: 2464-2467

17 Zhao J J, Zhuan C Q, Jiang X. Structure and mechanical properties of cubic $\mathrm{BC}_{2} \mathrm{~N}$ crystals within a random solid solution mode. Diam Relat Mater, 2010, 19: 1419-1422

18 Sun H, Jhi S H, Roundy D, et al. Structural forms of cubic BC ${ }_{2}$ N. Phys Rev B, 2001, 64: 094108

19 Guo X J, Liu Z Y, Luo X G, et al. Theoretical hardness of the cubic $\mathrm{BC}_{2} \mathrm{~N}$. Diam Relat Mater, 2007, 16: 526-530

20 Zhuang $\mathrm{C}$ Q, Zhao J J, Jiang X, et al. Structural stability, mechanical and electronic properties of cubic $\mathrm{BC}_{x} \mathrm{~N}$ crystals within a random solid solution model. J Phys Condens Mat, 2009, 21: 405401

21 Komatsu T, Nomura M, Kakudate $\mathrm{Y}$, et al. Synthesis and characterization of a shock-synthesized cubic B-C-N solid solution of composition $\mathrm{BC}_{2.5} \mathrm{~N}$. J Mater Chem, 1996, 6: 1799-1803

22 He J L, Tian Y J, Yu D L, et al. Orthorhombic $\mathrm{B}_{2} \mathrm{CN}$ crystal synthesized by high pressure and temperature. Chem Phys Lett, 2001, 340: $431-436$

23 Li Q, Zhou D, Wang H, et al. Crystal and electronic structures of superhard $\mathrm{B}_{2} \mathrm{CN}$ : An ab inition study. Solid State Commun, 2012, 152: $71-75$

24 Luo X G, Guo X J, Xu B, et al. Body-centered superhard $\mathrm{BC}_{2} \mathrm{~N}$ phases from first principles. Phys Rev B, 2007, 76: 3398-3407

25 Ribeiro F J, Tangney P, Louie S G, et al. Hypothetical hard structures of carbon with cubic symmetry. Phys Rev B, 2006, 74: 3840-3845

26 Hill R. The elastic behaviour of a crystalline aggregate. P Phys Soc Lond A, 1952, 65: 349-354

27 Voigt W. Lehrbuch der Kristallphysik: Teubner-Leipzig. New York: Macmillan, 1928

28 Reuss A, Angew Z. Calculation of the flow limits of mixed crystals on the basis of the plasticity of monocrystals. Z Angew Math Mech, 1929, 9: 49-58 
Application to TiSi 2 . J Appl Phys, 1998, 84: 4891-4904

31

$$
\text { 76-90 }
$$

39 Fletcher R. A new approach to variable metric algorithms. Comput J, 1970, 13: 317-322

40 Goldfarb D. A family of variable-metric methods derived by variational means. Math Comput, 1970, 24: 23-26

41 Shanno D F. Conditioning of quasi-Newton methods for function minimization. Math Comput, 1970, 24: 647-656

42 Oikawa K, Wulff L, Iijima T, et al. Promising ferromagnetic Ni-Co-Al shape memory alloy system. Appl Phys Lett, 2001, 79: 3290-3292

43 Zhang C L, Li J N, Han P D, et al. Study on alloying effects of $\mathrm{Ni}_{3} \mathrm{Al}$ with Cr Based on the pseudopotential plane-wave method (in Chinese). Rare Metal Mater Eng, 2008, 37: 1705-1709 [张彩丽，李晋敏，韩培德，等. Cr 对 $\mathrm{Ni}_{3} \mathrm{Al}$ 合金化效应的噟势平面波方法. 稀有 金属材料与工程, 2008, 37: 1705-1709]

44 Wu Z J, Zhao E J, Xiang H P, et al. Crystal structures and elastic properties of superhard $\operatorname{IrN}_{2}$ and $\operatorname{IrN}_{3}$ from first principles. Phys Rev B, 2007, 76: 054115

45 Levine J B, Tolbert S H, Kaner R B. Advancements in the search for superhard ultra-incompressible metal borides. Adv Funct Mater, 2009, 19: 3519-3533

46 Pugh S F. Relations between the elastic moduli and the plastic properties of polycrystalline pure metals. Philos Mag, 1954, 45: 823-843

47 Tian Y, Xu B, Zhao Z. Microscopic theory of hardness and design of novel superhard crystals. Int J Refract Met H, 2012, 33: 93-106

48 Chen X Q, Niu H, Li D, et al. Modeling hardness of polycrystalline materials and bulk metallic glasse. Intermetallics, 2011, 19: 1275-1281

49 Gao F M, He J L, Wu E D, et al. Hardness of covalent crystals. Phys Rev Lett, 2003, 91: 015502

50 Li F, Man Y H, Li C M, et al. Mechanical properties, minimum thermal conductivity, and anisotropy in bc-structure superhard materials. Comp Mater Sci, 2015, 102: 327-337

51 Wang J P, Li F, Ao J, et al. Investigation of elastic properties, hardness and thermal conductivity of new superhard material z-BC ${ }_{2} \mathrm{~N}$ (in Chinese). J Inorganic Mater, 2015, 30:467-473 [王军朋, 李凤, 敖靖, 等. 新型超硬材料 $\mathrm{z}-\mathrm{BC}_{2} \mathrm{~N}$ 的弹性, 硬度与热导率研究. 无机材 料学报, 2015, 30: 467-473]

52 Ranganathan S I, Starzewski M O. Universal elastic anisotropy index. Phys Rev Lett, 2008, 101: 055504

53 Chung D H, Buessem W R. The elastic anisotropy of crystal. J Appl Phys, 1967, 38: 2010-2012

54 ZhangY H, Franke P, SeifertH J, et al. Polymorphism of M3AlX Phases (M=Ti, Zr, Hf; X=C, N) and Thermomechanical Properties of Ti3AlN Polymorphs. J Am Ceram Soc, 2015, 98: 2570-2578 


\title{
Elasticity, hardness and anisotropies of bc- $\mathrm{B}_{n} \mathrm{CN}(n=1,2,4)$
}

\author{
HU Meng, CHEN ZhiQian*, LI ChunMei, LI Feng, WANG JunPeng \& JIAO LiNa \\ Faculty of Materials and Energy, Southwest University, Chongqing 400715, China
}

Based on the first-principles density functional theory with plane wave ultrasoft and norm-conserving pseudopotential methods, the elasticity and hardness of the three selected bc- $\mathrm{B}_{n} \mathrm{CN}(n=1,2,4)$ are investigated in details using LDA and GGA potential field. In addition, we pay close attention to their otherness of elastic and plastic anisotropies. The calculated results prove that these three kinds of structures are all stable in thermodynamics. Among these three structures, bc-BCN, which has the maximum absolute values of formation enthalpy and cohesive energy, is the most stable one with the lowest rate of atom $\mathrm{B}$. And the reason why bc-BCN is the most resisting structure and has the largest absolute value of energy is that it owns large number of relatively low energy bonds such as $\mathrm{B}-\mathrm{N}$, $\mathrm{B}-\mathrm{C}, \mathrm{C}-\mathrm{N}$ bonds and the fewest of $\mathrm{B}-\mathrm{B}$ bond. The analysis of elasticity and hardness results shows that the values of elastic modulus and hardness decrease accordingly with the increasing ratio of atom $\mathrm{B}$ in the system. Then in line with the universal elastic anisotropic index $A^{\mathrm{U}}$, percent elastic anisotropy $A_{\mathrm{B}}$ and $A_{\mathrm{G}}$, and shear anisotropic factors $A_{1}$, $A_{2}$ and $A_{3}$ of bc- $\mathrm{B}_{n} \mathrm{CN}(n=1,2,4)$, it is revealed that all of the three structures show different extent of anisotropy. Among these, bc-BCN displays better anisotropic property than c-BN as a whole. Finally, the ideal stress-strain curves of bc- $\mathrm{B}_{n} \mathrm{CN}(n=1,2,4)$ are fitted through the simulation of stepped tensile and shear deformation, and it indicates the anisotropy during deformation process. What's more, the matched curves reveal their respective yield surfaces, which are the easiest to slide. Additionally, the most effortless slip directions of different structures are deduced explicitly. In general, bc-BCN is the most easily to synthesize and most suitable for applications among these three compounds.

first-principles, elasticity, hardness, anisotropy

PACS: 71.15.Mb, 46.25.Cc, 62.20.Qp, 62.20.de

doi: $10.1360 /$ SSPMA2015-00315 